\title{
Vlasov description of the effects of nonlinear chromaticity on transverse coherent beam instabilities
}

\author{
M. Schenk, ${ }^{1,2, *}$ X. Buffat, ${ }^{1} \mathrm{~K} . \mathrm{Li},{ }^{1}$ and A. Maillard ${ }^{3}$ \\ ${ }^{1}$ European Organization for Nuclear Research (CERN), CH-1211 Geneva, Switzerland \\ ${ }^{2}$ École Polytechnique Fédérale de Lausanne (EPFL), CH-1015 Lausanne, Switzerland \\ ${ }^{3}$ École Normale Supérieure (ENS), F-75230 Paris, France
}

(Received 15 June 2018; published 24 August 2018)

\begin{abstract}
This paper extends the existing Vlasov theory for transverse coherent beam instabilities to account for nonlinear chromaticity. It confirms the hypothesis that nonlinear chromaticity has two effects on the beam dynamics: (i) it changes the effective impedance which results in a modification of the coherent frequencies of the head-tail modes, and (ii) it can introduce betatron detuning with longitudinal action and provide Landau damping of transverse modes. Based on the new formalism, these two mechanisms are identified and studied analytically with both an airbag and a Gaussian beam. To complete the study, the theory is successfully benchmarked against the circulant matrix solver BimBim as well as the PyHEADTAIL macroparticle tracking code.
\end{abstract}

DOI: 10.1103/PhysRevAccelBeams.21.084402

\section{INTRODUCTION}

The objective of this paper is to extend the Vlasov theory on impedance-driven transverse beam instabilities in proton synchrotrons to provide an analytical understanding of the coherent beam dynamics effects introduced by nonlinear chromaticity. The motivation for this study originates from the proposal of beam stabilization with a radio frequency (rf) quadrupole described and analyzed in detail in Refs. [1,2]. In summary, the purpose of the if quadrupole is to provide Landau damping in the transverse planes by producing an incoherent betatron frequency spread as a function of the longitudinal action of the particles in the bunch. This new method for Landau damping in the transverse planes is particularly promising in potential future colliders, e.g., the Future Circular Collider project [3], where traditional approaches such as magnetic octupoles (detuning with transverse action) experience a strongly reduced efficiency as a result of the high energy and low transverse emittances of the particle beams $[2,4]$.

It was shown in Ref. [5] that the beam dynamics effects of second-order chromaticity are equivalent to those of an rf quadrupole at first order. The major advantage of secondorder chromaticity over the rf quadrupole is that it can be enhanced and controlled in an existing machine without the

*michael.schenk@ cern.ch

Published by the American Physical Society under the terms of the Creative Commons Attribution 4.0 International license. Further distribution of this work must maintain attribution to the author(s) and the published article's title, journal citation, and DOI. need to develop and install additional equipment as demonstrated in the same reference. This makes it possible to experimentally validate numerical and analytical models describing the beam dynamics in presence of second-order chromaticity and is the reason why this paper focuses on nonlinear chromaticity rather than an rf quadrupole. Nevertheless, the analytical understanding gained of the effects of nonlinear chromaticity is directly applicable to an rf quadrupole.

Although the theory developed in this paper is valid for arbitrary orders, the second-order chromaticity is of particular interest here given the experimental observations made in the Large Hadron Collider (LHC) at CERN $[6,7]$. Both LHC measurements as well as tracking simulations indicate that second-order chromaticity introduces two beam dynamics effects: (i) it changes the effective impedance, i.e., the overlap sum of the beam spectrum with the impedance, leading to a modification of the transverse dipole modes and the associated coherent frequencies, and (ii) it introduces a betatron frequency spread as a function of longitudinal action which leads to a dispersion integral and Landau damping [8]. Indeed, the LHC experiments demonstrate that head-tail modes can be suppressed if the amount of second-order chromaticity is chosen accordingly. The same observations have been made also in simulations with an $\mathrm{rf}$ quadrupole [5]. In general, the two effects are interlinked and hence difficult to study separately, especially in particle tracking simulations. And, although they seem to govern the beam dynamics with second-order chromaticity as suggested by both experiments and simulations, the formal proof has yet been missing. The goal of this paper is to fill in the gaps from an analytical point-of-view. Specifically, the idea is to extend 
the existing Vlasov theory to include nonlinear chromaticities to identify and decouple the two effects analytically.

The paper is structured as follows. The first part of Sec. II reviews the basics of the Vlasov theory on transverse dipole modes in presence of linear chromaticity. Based on these derivations, the Vlasov formalism is extended to include arbitrary orders of chromaticity. The final result is an eigenvalue problem which is analytically solved for second-order chromaticity in Sec. III. This is done first for a longitudinal airbag beam which cannot experience Landau damping from nonlinear chromaticity, but whose dynamics is still affected by the change of the effective impedance. In the second part, arbitrary longitudinal phase space distributions are considered and a dispersion integral is derived under specific constraints on the shape of the impedance. The circulant matrix model (CMM), implemented in the BimBim code, is one of the two accelerator physics models employed in Sec. IV to benchmark the newly developed theory $[9,10]$. For further validation of the theory, results from PyHEADTAIL 6D macroparticle tracking simulations are included as well, both for airbag and Gaussian beams [11]. For the latter, stability diagram theory is compared to predictions from PyHEADTAIL for a specific head-tail mode driven by a highly narrow-band resonator. Finally, the main findings of the paper are summarized in Sec. V.

\section{VLASOV FORMALISM}

This section recaps the Vlasov formalism and introduces the notation used throughout the manuscript. The objectives are twofold. First, the Vlasov equation for transverse collective single-bunch dipole modes in a machine with purely linear chromaticity is recapped. Second, a newly developed theory is introduced which is valid also in presence of higher order chromaticity including dependencies of arbitrary orders in the relative momentum error. The conventions and notation used by A. Chao in Ref. [12] are closely followed to optimize readability and to make direct comparisons possible. All the derivations are made assuming the ultrarelativistic limit, i.e., $\beta=1$.

\section{A. Linear chromaticity}

Let $\Psi=\Psi\left(s, y, p_{y}, z, \delta\right)$ be the particle distribution of the bunch in $4 \mathrm{D}$ phase space, considering the longitudinal (2D) and only one transverse plane (2D). The parameter $s$ denotes the longitudinal position of the bunch along the accelerator and is equivalent to the time $t$ through $s=c t$, where $c$ is the speed of light. The variable pairs $\left(y, p_{y}\right)$ and $(z, \delta)$ correspond respectively to the transverse (horizontal or vertical) and longitudinal coordinates and conjugate momenta. These four phase space variables are all implicitly dependent on $s$. The Vlasov equation hence reads [12]

$$
\frac{d \Psi}{d s}=\left(\partial_{s}+y^{\prime} \partial_{y}+p_{y}^{\prime} \partial_{p_{y}}+z^{\prime} \partial_{z}+\delta^{\prime} \partial_{\delta}\right) \Psi=0,
$$

where $y^{\prime}, p_{y}^{\prime}, z^{\prime}$, and $\delta^{\prime}$ denote the first derivatives with respect to $s$. They are known from the transverse and longitudinal equations of motion describing the single particle beam dynamics (see, e.g., Ref. [12]). The impact of a transverse wakefield on the beam is accounted for by the $p_{y}^{\prime}$ term and is expressed in more detail in the following. The Fokker-Planck term describing, amongst others, radiation effects is neglected in our study as the focus is put on proton machines [13,14].

The Vlasov equation can be simplified using polar coordinates in the transverse and longitudinal planes

$$
\begin{aligned}
\left(y, p_{y}\right) & =\left(q \cos \theta,-\frac{\omega_{\beta, 0}}{c} q \sin \theta\right), \\
(z, \delta) & =\left(r \cos \phi, \frac{1}{\beta_{z}} r \sin \phi\right) .
\end{aligned}
$$

$\omega_{\beta, 0}$ denotes the unperturbed betatron frequency and $\beta_{z}=\eta c / \omega_{s}$ is the longitudinal Courant-Snyder beta function, where $\omega_{s}$ is the synchrotron frequency and $\eta$ the slip factor. Linear synchrotron motion is assumed throughout the manuscript. The Vlasov equation in polar coordinates reads

$$
\left(\partial_{s}+\frac{\omega_{\beta, 0}}{c}\left(1+\xi^{(1)} \delta\right) \partial_{\theta}+\frac{\omega_{s}}{c} \partial_{\phi}+\frac{F_{y}}{E} \partial_{p_{y}}\right) \Psi=0 .
$$

The betatron frequency is $\omega_{\beta}(\delta)=\left(1+\xi^{(1)} \delta\right) \omega_{\beta, 0}$, and $\xi^{(1)}=\left.\omega_{\beta, 0}^{-1}\left(\partial \omega_{\beta} / \partial \delta\right)\right|_{\delta=0}$ denotes the first-order chromaticity. $E=\gamma m_{0} c^{2}$ is the total energy of the beam particles, with $\gamma$ the Lorentz factor and $m_{0}$ the rest mass of the particles. $F_{y}$ describes the transverse force and represents here the effect of transverse dipolar wakefields on the beam. It is given by the convolution of the transverse wake with the longitudinal bunch profile, or, equivalently, by the overlap sum of the transverse impedance with the frequency spectrum of the bunch. As in Ref. [12], the longitudinal kick introduced by the transverse wakefield has been neglected since it has an insignificant impact on our studies. This is valid as long as synchro-betatron resonance conditions are avoided, and given that the transverse beam sizes remain small enough.

The solutions $\Psi$ to Eq. (3) are the collective transverse dipole modes. They can be described by a sum of a stationary solution and a perturbation term $\Psi=\Psi_{0}+\Psi_{1}$, with

$$
\begin{aligned}
& \Psi_{0}=g_{0}(r) f_{0}(q), \\
& \Psi_{1}=g_{1}(r, \phi) f_{1}(q, \theta) e^{-i \Omega s / c} .
\end{aligned}
$$

The longitudinal $g_{0}(r)$ and transverse $f_{0}(q)$ distributions being stationary can only have a radial dependence. The two planes are furthermore assumed to be fully decoupled which is why $\Psi_{0}$ can be expressed as a product of two 
independent functions. The perturbative distributions $g_{1}(r, \phi)$ and $f_{1}(q, \theta)$ represent respectively the longitudinal and transverse structures of the coherent mode. The exponential term describes its time evolution where $\Omega$ is the complex coherent frequency associated with the mode. The transverse instabilities under study are of dipolar nature and hence their transverse structure can be anticipated $f_{1}(q, \theta)=-D f_{0}^{\prime}(q) e^{i \theta} . D$ is the dipolar moment of the perturbed distribution and $f_{0}^{\prime}(q)$ is the first derivative of $f_{0}(q)$ with respect to $q$.

To further simplify the Vlasov equation, the wakefield force term is expressed in frequency domain using the transverse dipolar impedance $Z_{1}^{\perp}(\omega)$ and the frequency spectrum $\tilde{\rho}_{1}(\omega)$ of the bunch. The latter is given by the Fourier transform of $\Psi_{1}$ projected onto the $z$-axis

$$
\tilde{\rho}_{1}(\omega)=\frac{\omega_{s}}{\eta c} \int_{0}^{\infty} \int_{0}^{2 \pi} r e^{-\frac{i \omega}{c} r \cos \phi} g_{1}(r, \phi) d \phi d r .
$$

The wakefield force, including multiturn effects, is expressed as

$$
F_{y}=i \frac{D e^{2} \omega_{0}^{2}}{4 \pi^{2} c} e^{-i \Omega s / c} \sum_{p=-\infty}^{\infty} \tilde{\rho}_{1}\left(\omega^{\prime}\right) Z_{1}^{\perp}\left(\omega^{\prime}\right) e^{i \omega^{\prime} z / c},
$$

where $\omega^{\prime}=p \omega_{0}+\Omega$, with $\omega_{0}$ the angular revolution frequency of the bunch and $e$ the elementary charge. Equations (5) and (6) are inserted into Eq. (3) and all the partial derivatives, apart from $\partial_{\phi}$ can be evaluated. After linearization in the perturbation $\Psi_{1}$, the Vlasov equation reads

$$
\begin{aligned}
i[\Omega & \left.-\omega_{\beta, 0}\left(1+\xi^{(1)} \delta\right)-\omega_{s} \partial_{\phi}\right] g_{1}(r, \phi) \\
= & \frac{e^{2} \omega_{s}}{2 \omega_{\beta, 0} T_{0}^{2} E \eta} g_{0}(r) \sum_{p=-\infty}^{\infty} Z_{1}^{\perp}\left(\omega^{\prime}\right) \\
& \times \int_{0}^{\infty} \int_{0}^{2 \pi} \tilde{r} e^{\frac{i \omega^{\prime}}{c}(r \cos \phi-\tilde{r} \cos \tilde{\phi})} g_{1}(\tilde{r}, \tilde{\phi}) d \tilde{\phi} d \tilde{r},
\end{aligned}
$$

where $T_{0}=2 \pi / \omega_{0}$ is the revolution period of the beam.

To integrate the equation over $\phi, g_{1}(r, \phi)$ is decomposed into a series of azimuthal modes $g_{1}^{l}(r, \phi)$

$$
g_{1}(r, \phi)=\sum_{l^{\prime}=-\infty}^{\infty} R_{l^{\prime}}(r) e^{i l^{\prime} \phi} e^{i \xi^{(1)} \frac{\omega_{\beta, 0}, 0}{\eta c} \cos \phi},
$$

using the fact that $g_{1}(r, \phi)$ has a $2 \pi$-periodicity in $\phi$. The functions $g_{1}^{l}(r, \phi)$ are the eigenmodes of the free $\left(Z_{1}^{\perp} \equiv 0\right)$ Vlasov equation [Eq. (7)], where $l$ denotes the azimuthal mode number. The corresponding eigenvalues are $\Omega^{(l)}=\omega_{\beta, 0}+l \omega_{s}$, for $l \in \mathbb{Z}$. Plugging the decomposed $g_{1}(r, \phi)$ into Eq. (7) and after multiplying both sides with $e^{-i l \phi}$, one can perform the integration over $\phi$ and finds an infinite set of equations

$$
\begin{aligned}
& \left(\Omega^{(l)}-\omega_{\beta, 0}-l \omega_{s}\right) R_{l}(r) \\
& =i K g_{0}(r) \sum_{l, p=-\infty}^{\infty} \int_{0}^{\infty} r^{\prime} R_{l^{\prime}}\left(r^{\prime}\right) i^{l-l^{\prime}} Z_{1}^{\perp}\left(\omega^{\prime}\right) \\
& \quad \times J_{l}\left(\frac{\omega^{\prime}-\omega_{\xi}}{c} r\right) J_{l^{\prime}}\left(\frac{\omega^{\prime}-\omega_{\xi}}{c} r^{\prime}\right) d r^{\prime}, \quad \text { for } l \in \mathbb{Z} .
\end{aligned}
$$

$J_{l}(x)$ denotes the Bessel functions of the first kind. Furthermore, the definitions $\omega_{\xi}=\xi^{(1)} \omega_{\beta, 0} / \eta$ and $K=$ $\pi e^{2} \omega_{s} / \omega_{\beta, 0} T_{0}^{2} E \eta$ have been introduced. Finally, using the expression for $\Omega^{(l)}$, one obtains $\omega^{\prime}=p \omega_{0}+\omega_{\beta, 0}+l \omega_{s}$. The result in Eq. (9) is equivalent to Chao's Eq. (6.179) in Ref. [12].

\section{B. Higher order chromaticity}

The goal of this section is to introduce a general variation $\Delta \omega_{\beta}(\delta)$ of the betatron frequency with arbitrary orders of chromaticity $\xi^{(n)}$

$$
\Delta \omega_{\beta}(\delta)=\omega_{\beta, 0} \sum_{n=1}^{m} \frac{\xi^{(n)}}{n !} \delta^{n},
$$

with

$$
\xi^{(n)}=\left.\frac{1}{\omega_{\beta, 0}} \frac{\partial^{n} \omega_{\beta}}{\partial \delta^{n}}\right|_{\delta=0},
$$

and to evaluate the transverse beam stability in this case. The derivations explained in Eqs. (1) to (7) are analogous even for an arbitrary chromatic change of the betatron frequency. A dependency on higher order chromaticity can hence be introduced simply by replacing

$$
\omega_{\beta, 0}\left(1+\xi^{(1)} \delta\right) \rightarrow \omega_{\beta, 0}+\Delta \omega_{\beta}(\delta)
$$

on the left-hand side of Eq. (7). However, the eigenmode decomposition in Eq. (8) cannot be used anymore as an ansatz and needs to be replaced by a different one. It is convenient to define

$$
G_{1}(r, \phi) \doteq e^{\frac{i}{\omega_{s}} \int_{0}^{\phi} \Delta \omega_{\beta}(\delta(r, u)) d u} g_{1}(r, \phi)
$$

such that the Vlasov equation can be expressed as

$$
\begin{aligned}
{[\Omega-} & \left.\omega_{\beta, 0}+i \omega_{s} \partial_{\phi}\right] G_{1}(r, \phi) \\
= & -i K g_{0}(r) \sum_{p=-\infty}^{\infty} Z_{1}^{\perp}\left(\omega^{\prime}\right) \int_{0}^{\infty} \int_{0}^{2 \pi} \tilde{r} e^{i \frac{\omega^{\prime}}{c}(r \cos \phi-\tilde{r} \cos \tilde{\phi})} \\
& \times e^{\frac{i}{\omega_{s}} A(r, \phi, \tilde{r}, \tilde{\phi})} G_{1}(\tilde{r}, \tilde{\phi}) d \tilde{\phi} d \tilde{r},
\end{aligned}
$$

with 
$A(r, \phi, \tilde{r}, \tilde{\phi}) \doteq \int_{0}^{\phi} \Delta \omega_{\beta}(\delta(r, u)) d u-\int_{0}^{\tilde{\phi}} \Delta \omega_{\beta}(\delta(\tilde{r}, u)) d u$.

It is straightforward to show that Eq. (14) reduces to Eq. (7) when assuming a purely linear chromaticity $\Delta \omega_{\beta}=\omega_{\beta, 0} \xi^{(1)} \delta$.

In analogy to Sec. II A, the decomposition of $G_{1}(r, \phi)$ is chosen such that the functions $G_{1}^{l}(r, \phi)$ are the azimuthal eigenmodes of the free $\left(Z_{1}^{\perp} \equiv 0\right)$ Vlasov equation

$$
\left[\Omega-\omega_{\beta, 0}+i \omega_{s} \partial_{\phi}\right] G_{1}^{l}(r, \phi)=0 .
$$

By imposing again the $2 \pi$-periodicity in $\phi$ for $G_{1}(r, \phi)$, one finds the free eigenvalues and eigenmodes

$$
\begin{aligned}
\Omega^{(l)} & =\omega_{\beta, 0}+l \omega_{s}+\left\langle\Delta \omega_{\beta}\right\rangle_{\phi}, \\
G_{1}^{l}(r, \phi) & =R_{l}(r) e^{i\left(l+\frac{\left\langle\Delta \omega_{\beta}\right\rangle_{\phi}}{\omega_{s}}\right) \phi}, \quad \text { for } l \in \mathbb{Z} .
\end{aligned}
$$

The term $\left\langle\Delta \omega_{\beta}\right\rangle_{\phi}$ denotes the average betatron frequency change with respect to $\phi$ and is, in general, dependent on the longitudinal amplitude $r$ of every particle

$$
\left\langle\Delta \omega_{\beta}\right\rangle_{\phi}(r)=\frac{1}{2 \pi} \int_{0}^{2 \pi} \Delta \omega_{\beta}(\delta(r, \phi)) d \phi .
$$

It describes the betatron frequency spread introduced through detuning with longitudinal amplitude. It will become clear in the following that this term leads to Landau damping. One can already see that for odd orders of chromaticity $\xi^{(2 n+1)}, n \in \mathbb{N}_{0}$ the average frequency spread vanishes $\left\langle\Delta \omega_{\beta}\right\rangle_{\phi}(r) \equiv 0$. This result is independent of the longitudinal particle distribution. Hence, odd orders of chromaticity do not introduce Landau damping of slow head-tail modes that build up over several synchrotron periods. On the other hand, even orders $\xi^{(2 n)}, n \in \mathbb{N}$ of chromaticity introduce a betatron frequency spread with longitudinal amplitude that does not average out over time, which leads to Landau damping. In either case, though, both odd and even orders of chromaticity introduce a change of the effective impedance and modify the coherent frequencies of the modes. Both effects will be demonstrated and discussed in Sec. III.

Using a series of the free eigenmodes [Eq. (17)] as an ansatz for $G_{1}(r, \phi)$ in Eq. (14), one obtains

$$
\begin{aligned}
& \sum_{l^{\prime \prime}=-\infty}^{\infty} R_{l^{\prime \prime}}(r)\left(\Omega-\omega_{\beta, 0}-l^{\prime \prime} \omega_{s}-\left\langle\Delta \omega_{\beta}\right\rangle_{\phi}(r)\right) e^{i l^{\prime \prime} \phi} \\
& =-i K g_{0}(r) \sum_{l^{\prime}, p=-\infty}^{\infty} Z_{1}^{\perp}\left(\omega^{\prime}\right) \int_{0}^{\infty} \int_{0}^{2 \pi} \tilde{r} e^{\frac{i \omega^{\prime}}{c}[r \cos \phi-\tilde{r} \cos \tilde{\phi}]} \\
& \quad \times R_{l^{\prime}}(\tilde{r}) e^{i l^{\prime} \tilde{\phi}} e^{\frac{i}{\omega_{s}}[B(r, \phi)-B(\tilde{r}, \tilde{\phi})]} d \tilde{\phi} d \tilde{r}
\end{aligned}
$$

where

$$
B(r, \phi) \doteq \int_{0}^{\phi}\left[\Delta \omega_{\beta}(\delta(r, u))-\left\langle\Delta \omega_{\beta}\right\rangle_{\phi}(r)\right] d u .
$$

The next step is to multiply both sides with $e^{-i l \phi}$ and to perform the integration over $\phi$ from 0 to $2 \pi$

$$
\begin{aligned}
& {\left[\Omega^{(l)}-\omega_{\beta, 0}-l \omega_{s}-\left\langle\Delta \omega_{\beta}\right\rangle_{\phi}(r)\right] R_{l}(r)} \\
& =-i 2 \pi K g_{0}(r) \sum_{l^{\prime}, p^{\prime}=-\infty}^{\infty} Z_{1}^{\perp}\left(\omega^{\prime}\right) \\
& \quad \times \int_{0}^{\infty} \tilde{r} R_{l^{\prime}}(\tilde{r}) \overline{H_{l}^{p^{\prime}}(r)} H_{l^{\prime}}^{p^{\prime}}(\tilde{r}) d \tilde{r}, \quad \text { for } l \in \mathbb{Z},
\end{aligned}
$$

where

$$
H_{l}^{p}(r) \doteq \frac{1}{2 \pi} \int_{0}^{2 \pi} e^{i l \phi} e^{-\frac{i \omega^{\prime}}{c} r \cos \phi} e^{-\frac{i}{\omega_{s}} B(r, \phi)} d \phi,
$$

with $l, p \in \mathbb{Z} . H_{l}^{p}(r)$ can be perceived as a generalized Bessel function. It is easy to show that in the event of a purely linear chromaticity, $H_{l}^{p}(r)$ reduces to the Bessel function of the first kind (see Appendix A). The phase terms $e^{-i B(r, \phi) / \omega_{s}}$ describe the alteration of the interaction of the beam with the impedance caused by arbitrary orders of chromaticity. The result is that the overlap sum over index $p^{\prime}$ in Eq. (21) between the $H_{l}^{p^{\prime}}(r)$ functions and the impedance $Z_{1}^{\perp}\left(\omega^{\prime}\right)$ changes. In time domain the chromatic phase terms can be understood as a change of the synchronicity between wake kicks, betatron, and synchrotron motion of the particles. This causes a change of the coherent frequency $\Omega$, both for the real and imaginary (instability rise time) components, of all the modes and is studied in more detail in Secs. III and IV for specific beam models. This mechanism can mimic the "stabilization" of a given coherent mode, if the change of effective impedance induced by a change of chromaticity is such that the growth rate of the particular mode is reduced. However, the modification of the effective impedance does not introduce a frequency spread and there is no increase of the area of stability in the complex frequency space. Hence, this effect is not related to Landau damping. Nevertheless, Landau damping is still part of the beam dynamics with higher order chromaticity. It is introduced through $\left\langle\Delta \omega_{\beta}\right\rangle_{\phi}(r) \neq 0$ from even orders of chromaticity. This term leads to a dispersion integral and to an increase of the area of stability in the complex frequency space as shown in the following. To summarize, Eq. (21) clearly decouples the two beam dynamics effects introduced by higher order chromaticity.

To further simplify Eq. (21), it can be multiplied with $r H_{l}^{p}(r)$ and integrated over $r$ from 0 to $\infty$ 


$$
\begin{aligned}
\sigma_{l p}= & -i K \sum_{l^{\prime}, p^{\prime}=-\infty}^{\infty} \sigma_{l^{\prime} p^{\prime}} Z_{1}^{\perp}\left(p^{\prime} \omega_{0}+\Omega^{(l)}\right) \\
& \times \int_{0}^{\infty} \frac{r g_{0}(r) \overline{H_{l}^{p^{\prime}}}(r) H_{l}^{p}(r)}{\Omega^{(l)}-\omega_{\beta, 0}-l \omega_{s}-\left\langle\Delta \omega_{\beta}\right\rangle_{\phi}(r)} d r,
\end{aligned}
$$

where

$$
\sigma_{l p} \doteq \int_{0}^{\infty} r R_{l}(r) H_{l}^{p}(r) d r .
$$

When neglecting azimuthal mode interactions (weak-wake approximation), the summation on $l^{\prime}$ can be ignored and one ends up with an infinite set of separate equations, one for each azimuthal mode. The dispersion integral on the right hand side of Eq. (23) indicates that the term $\left\langle\Delta \omega_{\beta}\right\rangle_{\phi}(r)$ in the denominator indeed introduces Landau damping. A dispersion relation that makes the computation of stability boundary diagrams with nonlinear chromaticity possible is derived in Sec. III B and numerical solutions are shown in Sec. IV.

\section{SOLUTIONS OF THE VLASOV EQUATION WITH NONLINEAR CHROMATICITY}

In this section, solutions of the previously derived Vlasov equation (23) are determined for two different types of longitudinal particle distributions. The first type is an airbag beam for which all the particles have exactly the same longitudinal amplitude. As a result, there can be no net betatron frequency spread from chromaticity of any order $\left(\left\langle\Delta \omega_{\beta}\right\rangle_{\phi}(r) \equiv 0\right)$ and hence there is no Landau damping. For that case, an exact solution of Eq. (23) can be determined for arbitrary orders of chromaticity. The second type of longitudinal distributions is general as it accounts for a spread in the longitudinal action of the particles, such as Gaussian and waterbag beams. In that case, Eq. (23) cannot be solved exactly. Instead, the analytical solution is determined with strict assumptions on the shape of the transverse dipolar impedance $Z_{1}^{\perp}(\omega)$ (narrow-band resonator).

The two types of distributions are chosen to decouple the two beam dynamics effects that have been identified in Sec. II B. Nonlinear chromaticity cannot introduce Landau damping for an airbag beam, but it does cause a change of effective impedance. Hence, for an airbag model, the latter mechanism can be studied independently of the Landau damping which facilitates the understanding of the beam dynamics in presence of higher order chromaticity in general. With the second type of beams on the other hand, there is an interplay of both mechanisms as shown by Eq. (21). The analytical results derived and presented here are benchmarked numerically with two different accelerator physics models in Sec. IV.

\section{A. Airbag model}

In case of an airbag model, the beam particles populate an infinitesimally thin elliptical shell in longitudinal phase space. Such a distribution is also known as a hollow beam model [12]. It is described by

$$
g_{0}(r)=\frac{N \eta c}{2 \pi \omega_{s} \hat{z}} \boldsymbol{\delta}(r-\hat{z})
$$

where $N$ is the bunch intensity in number of particles, $\hat{z}$ defines the radius of the distribution in normalized longitudinal phase space $\left(z, \beta_{z} \delta\right)$, and $\boldsymbol{\delta}$ is the Dirac delta function. For the airbag beam, all the particles have the same longitudinal action $J_{z}=\hat{z}^{2} / 2 \beta_{z}$. An illustration is displayed in Fig. 1.

In the weak-wake approximation considered here, azimuthal mode coupling can be neglected, meaning that the summation over index $l^{\prime}$ can be ignored in Eq. (23). Instead, one can solve the equations for all the azimuthal modes independently of one another. Using the airbag distribution, the integration over $r$ can be easily performed leading to

$$
\begin{aligned}
\sigma_{l p}= & -i \frac{N e^{2} c}{2 \omega_{\beta, 0} T_{0}^{2} E} \sum_{p^{\prime}=-\infty}^{\infty} \sigma_{l p^{\prime}} Z_{1}^{\perp}\left(\omega^{\prime}\right) \\
& \times \frac{\overline{H_{l}^{p^{\prime}}}(\hat{z}) H_{l}^{p}(\hat{z})}{\Omega^{(l)}-\omega_{\beta, 0}-l \omega_{s}-\left\langle\Delta \omega_{\beta}\right\rangle_{\phi}(\hat{z})} .
\end{aligned}
$$

The detuning term $\left\langle\Delta \omega_{\beta}\right\rangle_{\phi}(\hat{z})=$ const. is now independent of the longitudinal amplitude $r$ and is identical for all the particles. As expected, the dispersion integral has disappeared from the equation which can be interpreted as the absence of Landau damping.

The eigenvalue problem in Eq. (26) can be analytically solved and the solution reads

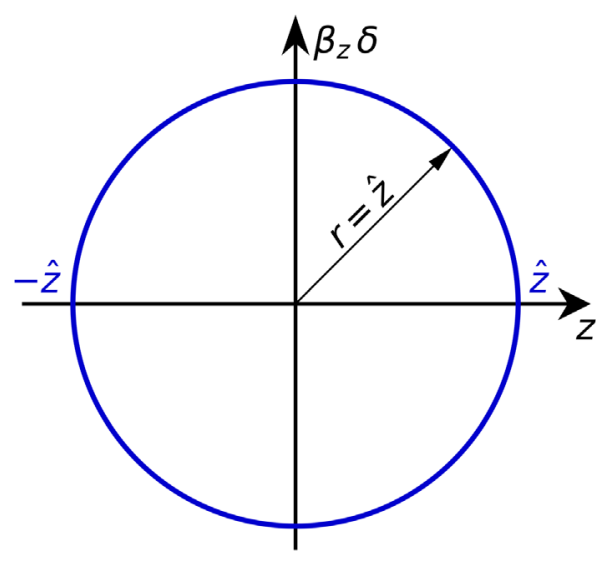

FIG. 1. Illustration of the airbag model in normalized longitudinal phase space. The particles populate a circle of radius $r=\hat{z}$ (blue). 


$$
\begin{aligned}
\Omega^{(l)} & -\omega_{\beta, 0}-l \omega_{s}-\left\langle\Delta \omega_{\beta}\right\rangle_{\phi}(\hat{z}) \\
& =-i \frac{N e^{2} c}{2 \omega_{\beta, 0} T_{0}^{2} E} \sum_{p=-\infty}^{\infty} Z_{1}^{\perp}\left(p \omega_{0}+\omega_{\beta, 0}+l \omega_{s}\right)\left|H_{l}^{p}(\hat{z})\right|^{2},
\end{aligned}
$$

where $\omega^{\prime} \approx p \omega_{0}+\omega_{\beta, 0}+l \omega_{s}$ and $l \in \mathbb{Z}$. We have obtained an explicit expression for the coherent frequency of each azimuthal mode $l$. One feature that is discussed further in Sec. IV is that the average detuning term $\left\langle\Delta \omega_{\beta}\right\rangle_{\phi}(\hat{z})$ only affects the real part of $\Omega^{(l)}$ and is identical for all the azimuthal modes.

Equation (27) is a generalization of Chao's Eq. (6.188) derived in Ref. [12] and is valid for arbitrary orders of chromaticity. It reduces to Chao's equation for a purely linear chromaticity as shown in Appendix A.

\section{B. Arbitrary longitudinal distributions}

The goal is now to study the eigenvalue problem in Eq. (23) for arbitrary longitudinal distributions $g_{0}(r)$, for which, in general, there is a spread in amplitudes $r$ (or longitudinal action $J_{z}$ ) among the particles within the bunch. In combination with even orders of chromaticity, the spread in action translates into an amplitude-dependent betatron frequency distribution $\left\langle\Delta \omega_{\beta}\right\rangle_{\phi}(r)$ and Landau damping. This can be seen from the dispersion integral on the right hand side of Eq. (23). This is similar to a dispersion integral for detuning with transverse amplitude as introduced for instance by magnetic octupoles. Both detuning with longitudinal and transverse amplitude lead to an increase of the area of stability in the complex frequency space [8].

The eigenvalue problem for arbitrary distributions is difficult to solve, even numerically. Hence, to obtain an analytical solution where the dispersion relation and Landau damping become even more apparent, and can be benchmarked against numerical models, constraints are imposed on the shape of the impedance. Here, a highly narrow-band resonator impedance is considered, such that effectively

$$
Z_{1}^{\perp}\left(\omega^{\prime}\right)= \begin{cases}Z_{p_{0}} \neq 0, & p=p_{0}, \\ 0, & \text { otherwise. }\end{cases}
$$

For this type of impedance, Eq. (23) simplifies to

$$
1=-i K Z_{p_{0}} \int_{0}^{\infty} \frac{r g_{0}(r)\left|H_{l}^{p_{0}}(r)\right|^{2}}{\Omega^{(l)}-\omega_{\beta}(r)-l \omega_{s}} d r
$$

where $\omega_{\beta}(r)=\omega_{\beta, 0}+\left\langle\Delta \omega_{\beta}\right\rangle_{\phi}$. Equation (29) is a dispersion relation. To obtain the stability boundary diagram, one considers the coherent frequency $\Omega_{\text {lin }}^{(l)}$ in absence of Landau damping (linear lattice). This can be achieved by ignoring the frequency spread, i.e., by setting $\left\langle\Delta \omega_{\beta}\right\rangle_{\phi}(r)=0$ in Eq. (29)

$$
1=-i \frac{K Z_{p_{0}}}{\Omega_{\operatorname{lin}}^{(l)}-\omega_{\beta, 0}-l \omega_{s}} \int_{0}^{\infty} r g_{0}(r)\left|H_{l}^{p_{0}}(r)\right|^{2} d r .
$$

The integral over $r$ can be easily performed numerically. Combining Eqs. (29) and (30), one finds the formula to determine the stability boundary diagrams

$$
\begin{aligned}
\left(\Delta \Omega_{\text {lin }}^{(l)}\right)^{-1} & =\frac{1}{\mathcal{N}} \int_{0}^{\infty} \frac{r g_{0}(r)\left|H_{l}^{p_{0}}(r)\right|^{2}}{\Omega^{(l)}-\omega_{\beta}(r)-l \omega_{s}} d r, \\
\mathcal{N} & =\int_{0}^{\infty} r g_{0}(r)\left|H_{l}^{p_{0}}(r)\right|^{2} d r,
\end{aligned}
$$

where $\Delta \Omega_{\text {lin }}^{(l)}=\Omega_{\text {lin }}^{(l)}-\omega_{\beta, 0}-l \omega_{s}$. The stability boundary diagram is obtained by computing the frequency shifts $\Delta \Omega_{\text {lin }}^{(l)}$ for different values of $\Omega^{(l)}$. According to the Landau bypass rule, a small complex part $i \varepsilon$ is added to the denominator of Eq. (31) to perform the integration [15]. An example for Gaussian beams is discussed in Sec. IV.

By making additional assumptions on the beam spectrum and impedance, it can be shown that the dispersion relation derived here is equivalent to the results found by Scott Berg and Ruggiero, originally presented in Ref. [8]. The proof is written out in Appendix B.

\section{BENCHMARKS}

This section summarizes the benchmarks that were made to validate the new formalism. Two different accelerator physics models are employed-a circulant matrix solver called BimBim and the PyHEADTAIL 6D macroparticle tracking code. For the sake of simplicity the tests only go up to order two in chromaticity, but extending them for arbitrary orders is straightforward with the formalism provided in Sec. II B. After introducing the tools and methods used for the comparison with analytical calculations, the new Vlasov formalism is tested first for an airbag model without Landau damping, and subsequently for a Gaussian beam which also experiences Landau damping.

\section{A. Tools and methods}

PyHEADTAIL is a tracking code developed at CERN and is the successor of the HEADTAIL program [16]. A detailed description of the model and available features is given in Ref. [11]. PyHEADTAIL being a macroparticle tracking code, does not directly compute the coherent frequency shifts of the head-tail modes, but rather it outputs the bunch centroid signal in time domain. In the event of an instability, the amplitude of the bunch centroid oscillation rises exponentially. After a certain latency time, which depends amongst others on the initial particle distribution, there is usually one dominant (aka. most unstable) mode 
that establishes and is responsible for the amplitude growth. The growth rate of the exponential hence corresponds to the imaginary part of the coherent frequency shift of the most unstable mode. The other head-tail modes that are present, but possibly stable or at least have a much slower rise time, cannot be accessed through time domain analysis. One method to obtain the imaginary frequency shifts of individual azimuthal modes is to analyze the growth rates of each synchrotron side band in frequency domain, using a sliding window Fourier transform. With this technique, stable modes can in principle also be made available by exciting them and measuring their rate of decay. For the studies presented here, both time- and frequency domain analysis methods have been employed for comparison, and an excellent agreement was always found on the growth rate of the most unstable mode. In the following, however, only the results from the time domain analysis are shown. To obtain the real part of the coherent frequency shift, a Fourier transform (Fast Fourier Transform or Sussix [17]) of the centroid motion is used. The shift is measured with respect to the unstable synchrotron side band.

BimBim is another accelerator physics code developed at CERN and its main purpose is to model beam-beam and wakefield effects. The underlying scheme is the circulant matrix model described in Refs. $[9,10]$. BimBim solves the circulant matrix and outputs directly the complex coherent frequency shifts of "all the possible" (stable and unstable) modes. Naturally, the chosen number of slices and rings for the discretization of the longitudinal phase space puts a limit on the maximum order of the unstable mode that can be described by the model. Since the formalism is based on matrices, it only accounts for linear dependencies on the transverse phase space variables, and hence it cannot model, for instance, detuning with transverse amplitude as introduced by Landau octupoles. In the longitudinal plane, however, nonlinear dependencies are correctly described and hence arbitrary orders of chromaticity can be included. Another important limitation of the code is that it assumes the wakefield to decay within one turn. While this is a valid approximation for broad-band resonator impedances, it is no longer fulfilled for narrow-band resonators with large quality factors. For the latter, the wakefields typically keep ringing for several revolution periods.

\section{B. Airbag model}

\section{Linear chromaticity}

To ensure that the new formalism works properly numerically, it is benchmarked first against the well-known case of an airbag model assuming a purely linear chromaticity. In that case,

$$
\begin{aligned}
\left\langle\Delta \omega_{\beta}\right\rangle_{\phi}(r) & =0, \\
B(r, \phi) & =\xi^{(1)} \frac{\omega_{\beta, 0} r}{\beta_{z}}(1-\cos \phi),
\end{aligned}
$$

and Eq. (27) reduces to Chao's Eq. (6.188) in Ref. [12] as proven in Appendix A. This expression allows to analytically compute the complex coherent frequency shifts of each azimuthal mode. The infinite sum over index $p$ is approximated numerically by a sum extending from $p=$ $-10^{5}$ to $10^{5}$. This was found to be enough to achieve convergence for the specific case discussed here.

Equation (27) is benchmarked against BimBim and PyHEADTAIL using the machine and beam parameters listed in Table I. The values are loosely based on the CERN Super Proton Synchrotron at injection energy. The machine impedance has been replaced by a broad-band $(Q=1)$ resonator. For BimBim, using one ring for the discretization of the longitudinal phase space is what is required to model an airbag beam. Furthermore, 90 azimuthal slices were found to be enough for the results to converge, given the low order of the modes that are considered. For PyHEADTAIL, $N_{\mathrm{mp}}=5 \times 10^{5}$ macroparticles were tracked over $N_{\text {turns }}=10^{5}$ turns. The results are summarized in Fig. 2. The analytical calculations are given by the colored lines and represent the real (upper plot) and imaginary (lower plot) coherent frequency shifts of the six lowest order azimuthal modes as a function of linear chromaticity $\xi^{(1)}$. The real part is measured with respect to the unstable synchrotron side band $l$, i.e., $\operatorname{Re} \Delta \Omega^{(l)}=\operatorname{Re}\left(\Omega^{(l)}-\omega_{\beta, 0}-l \omega_{s}\right)$, to improve the readability of the plot. It can be seen that the azimuthal modes are degenerate, i.e., the modes for a specific positive and negative azimuthal number are identical. This will no longer be the case when introducing second-order chromaticity as described below. As expected, for $\xi^{(1)}<0$ the most unstable mode is a head-tail mode zero. For increasing

TABLE I. Main parameters used in PyHEADTAIL and BimBim simulations to benchmark the Vlasov formalism for an airbag beam. The parameters $N_{\text {mp }}$ and $N_{\text {turns }}$ are relevant exclusively for the PyHEADTAIL simulations, while $N_{\text {rings }}$ and $N_{\text {slices }}$ are valid solely for the calculations with BimBim.

\begin{tabular}{lcc}
\hline \hline Parameter & & Value \\
\hline Relativistic gamma & $\gamma$ & 27.7 \\
Synchrotron tune & $Q_{s}$ & 0.017 \\
Longitudinal beta function & $\beta_{z}$ & $115 \mathrm{~m}$ \\
Bunch intensity & $N$ & $10^{9} \mathrm{p}$ \\
Longitudinal action & $J_{z}$ & $3 \times 10^{-4} \mathrm{~m}$ \\
Resonator shunt impedance & $R_{s}$ & $10^{7} \Omega / \mathrm{m}$ \\
Resonator frequency & $f_{r}$ & $0.8 \mathrm{GHz}$ \\
Resonator quality factor & $Q$ & 1 \\
Wake decay time & $N_{\text {wake }}$ & 1 turn \\
Number of $p$ modes & $N_{p}$ & $2 \times 10^{5}$ \\
Number of macroparticles & $N_{\text {mp }}$ & $5 \times 10^{5} \mathrm{p}$ \\
Number of turns & $N_{\text {turns }}$ & $10^{5}$ turns \\
Number of rings & $N_{\text {rings }}$ & 1 \\
Number of azimuthal slices & $N_{\text {slices }}$ & 90 \\
\hline \hline
\end{tabular}




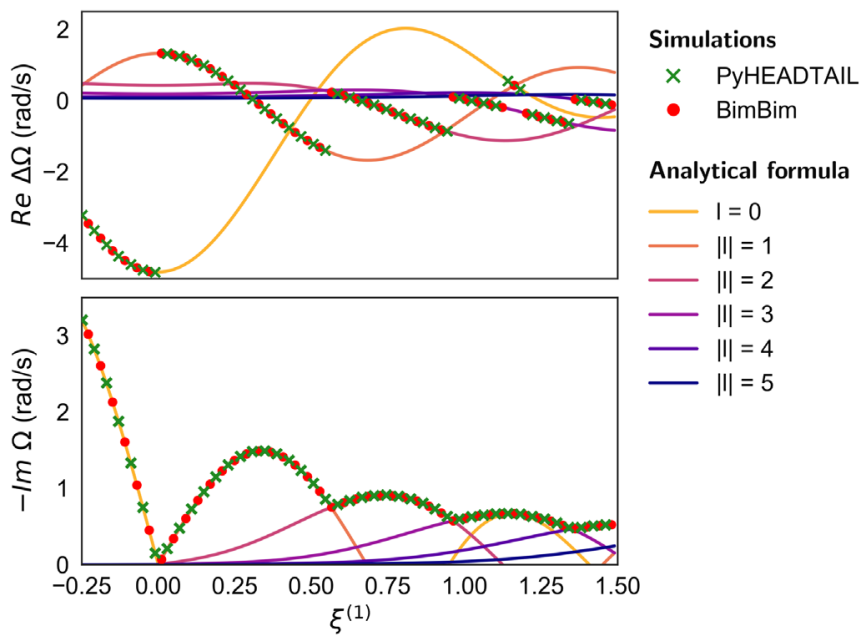

FIG. 2. Real (top) and imaginary (bottom) coherent frequency shifts as a function of purely linear chromaticity $\xi^{(1)}$ for an airbag model with machine and beam parameters listed in Table I. Results from BimBim (red circles), PyHEADTAIL simulations analyzed with Sussix (green crosses), and analytical calculations (solid lines) are shown.

$\xi^{(1)}>0$, the most unstable mode changes from azimuthal mode one through five given the limited range of chromaticity. The locations of the transitions between the modes depend on the choice of beam and impedance parameters. The outputs from BimBim (red dots) and PyHEADTAIL (green crosses) after post-processing are shown on top of the analytical results and prove an excellent agreement with the most unstable mode at each value of chromaticity. Not only is the mode number the same, but both the real and imaginary parts are in perfect agreement between the three approaches. The fact that two different numerical accelerator physics models confirm the theory to that degree of accuracy is very satisfying and proves that the analytical description works well for a basic linear case.

\section{Second-order chromaticity}

In the following, nonzero first- and second-order chromaticities are assumed. In that case, the change of betatron frequency is given by $\Delta \omega_{\beta}=\omega_{\beta, 0}\left(\xi^{(1)} \delta+\xi^{(2)} \delta^{2} / 2\right)$. Hence,

$$
\begin{aligned}
\left\langle\Delta \omega_{\beta}\right\rangle_{\phi}(r)= & \xi^{(2)} \frac{\omega_{\beta, 0} r^{2}}{4 \beta_{z}^{2}}, \\
B(r, \phi)= & \xi^{(1)} \frac{\omega_{\beta, 0} r}{\beta_{z}}(1-\cos \phi) \\
& -\xi^{(2)} \frac{\omega_{\beta, 0} r^{2}}{4 \beta_{z}^{2}} \sin \phi \cos \phi .
\end{aligned}
$$

Combining Eqs. (27) and (33), one obtains again an explicit expression for the coherent frequency shifts for each azimuthal mode. The same number of $p$ modes is used

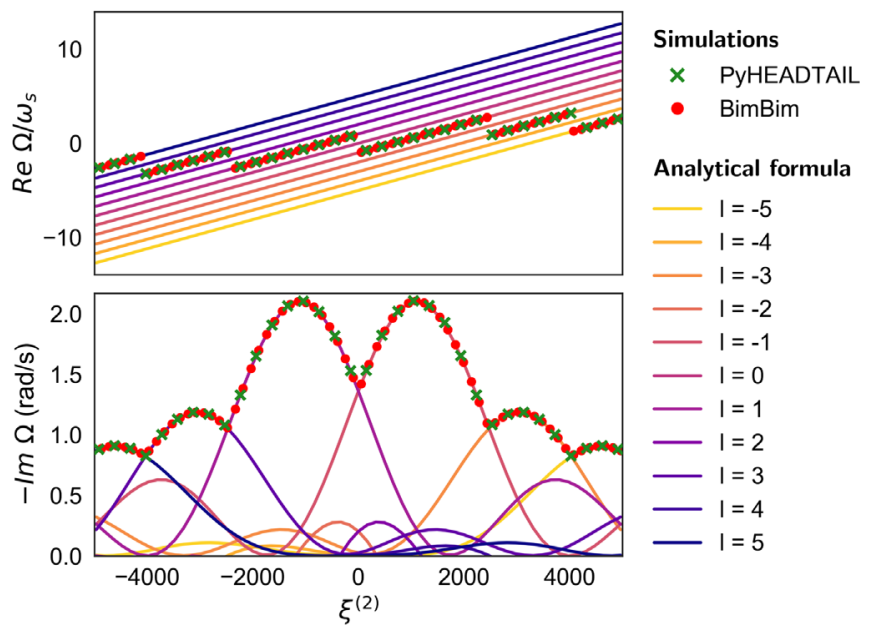

FIG. 3. Real (top) and imaginary (bottom) coherent frequencies as a function of second-order chromaticity $\xi^{(2)}$ at fixed $\xi^{(1)}=$ 0.25 for an airbag model with machine and beam parameters listed in Table I. Results from the circulant matrix model BimBim (red circles), PyHEADTAIL simulations analyzed with Sussix (green crosses), and analytical calculations (solid lines) are shown.

to guarantee the convergence of the overlap sum. Analytical results for the same machine and beam parameters as above (Table I) are plotted in Fig. 3 and are represented by the colored lines. The first azimuthal modes up to order five are shown. Note that in this figure the real part is normalized to the synchrotron frequency and the vertical axis hence corresponds to the azimuthal mode number. One can see that $\xi^{(2)}$ indeed changes the effective impedance, and eventually, transitions to other, now more unstable, azimuthal modes occur. This is very similar to the case of first-order chromaticity. A major difference with respect to Fig. 2, however, is that the degeneracy in the azimuthal mode number is lifted. For a certain absolute value of the mode number $l$, the modes with the two opposite signs are no longer identical. Another observation is that only every other mode is excited (here only odd mode numbers). Additionally, the real part of the coherent frequency shift is dominated by the constant and realvalued $\left\langle\Delta \omega_{\beta}\right\rangle_{\phi}(\hat{z})$, which is the same for all the azimuthal modes as illustrated by Fig. 3 (top). This is specific to the airbag beam, and is again a result of the absence of a spread in longitudinal amplitude. For PyHEADTAIL and BimBim again only the most unstable modes are shown with their real and imaginary parts for each value of $\xi^{(2)}$. As in the linear chromaticity case, the theoretical predictions are again in perfect agreement with both the tracking and circulant matrix models, which confirms that the formalism developed in Sec. II B is indeed valid for the airbag beam.

To summarize, the new theory describes the change of effective impedance from nonlinear chromaticity very accurately and gives satisfying results for the airbag model. The next step is to introduce a particle distribution that has a 


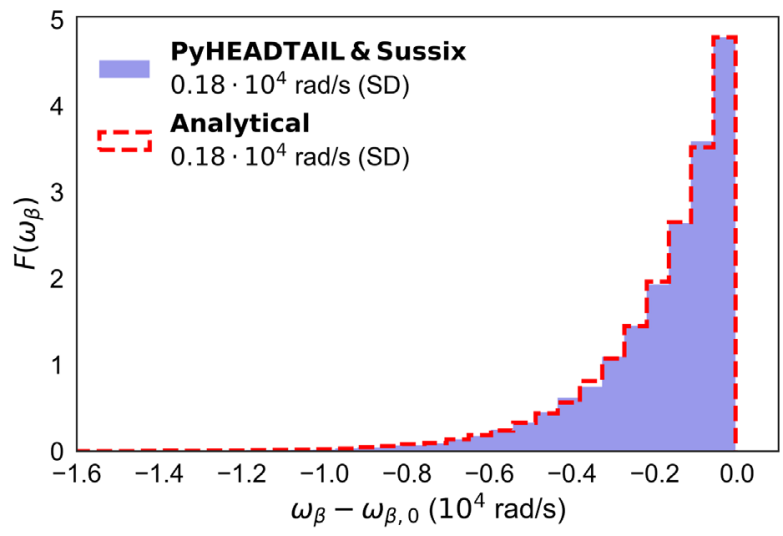

FIG. 4. Comparison of the betatron frequency distributions obtained using analytical formula (red dashed bars) and PyHEADTAIL in combination with a Sussix frequency analysis (blue area). The standard deviation (SD) is also listed for both cases.

spread in longitudinal amplitudes to validate the theory also in presence of Landau damping.

\section{Gaussian beam}

To study the effect of Landau damping, a Gaussian beam is used for the comparison between the theory and the tracking model

$$
g_{0}(r)=\frac{N \eta c}{2 \pi \sigma_{z}^{2} \omega_{s}} e^{-r^{2} / 2 \sigma_{z}^{2}}
$$

where $\sigma_{z}$ denotes the one-sigma bunch length.

For the first part of the validation procedure, the betatron frequency distributions introduced by a specific amount of $\xi^{(2)}\left(\right.$ at $\left.\xi^{(1)}=0\right)$ are compared between analytical formula and PyHEADTAIL. Figure 4 summarizes the results. The blue histogram corresponds to the output from the tracking simulations of $3 \times 10^{4}$ macroparticles over ten synchrotron periods using the machine parameters listed in Table II and neglecting the effect of the impedance. The betatron frequencies of every particle have been analyzed with Sussix. The red dashed bar diagram corresponds to the analytical calculation obtained using $\left\langle\Delta \omega_{\beta}\right\rangle_{\phi}(r)$ with $g_{0}(r)$ from Eq. (34). The agreement between the tracking model and the analytical formula is excellent and confirms the correct implementation of the detuning from second-order chromaticity in PyHEADTAIL.

Based on this result, the next step is to design a study case which fulfils the approximations and assumptions made when deriving the dispersion relation in Eq. (31). The main one was to use a highly narrow-band resonator impedance as defined in Eq. (28) such that there would be only one dominant term in the sum over index $p$ in Eq. (23). This can be achieved by tuning the quality factor and the frequency of the resonator accordingly. The optimization
TABLE II. Main parameters used in PyHEADTAIL to benchmark the Vlasov formalism for a Gaussian beam. The resonator impedance is highly narrow-band and its frequency has been tuned well to ensure that the overlap with the beam spectrum is largest at one specific mode $p_{0}$ and negligible everywhere else.

\begin{tabular}{lcc}
\hline \hline Parameter & & Value \\
\hline Relativistic gamma & $\gamma$ & 27.7 \\
Synchrotron tune & $Q_{s}$ & 0.017 \\
Longitudinal beta function & $\beta_{z}$ & $115 \mathrm{~m}$ \\
Bunch intensity & $N$ & $10^{9} \mathrm{p}$ \\
Bunch length & $\sigma_{z}$ & $0.21 \mathrm{~m}$ \\
Resonator shunt impedance & $R_{s}$ & $5 \times 10^{12} \Omega / \mathrm{m}$ \\
Resonator frequency & $f_{r}$ & $0.7993 \mathrm{GHz}$ \\
Resonator quality factor & $Q$ & $5 \times 10^{4}$ \\
Wake decay time & $N_{\text {wake }}$ & 80 turns \\
Number of $p$ modes & $N_{p}$ & 1 \\
Number of macroparticles & $N_{\mathrm{mp}}$ & $5 \times 10^{5} \mathrm{p}$ \\
Number of turns & $N_{\text {turns }}$ & $10^{5}$ turns \\
\hline \hline
\end{tabular}

was done by looking at the overlap area between the impedance and the bunch spectra $\left(H_{l}^{p}(r)\right.$ functions) for the main lowest-order modes. The resonator frequency and quality factor were tuned to match the spectral maximum of the azimuthal mode zero while remaining small for all the other modes. The final values are listed in Table II.

To verify that the single-peak approximation is valid for the chosen impedance, a scan in linear chromaticity was performed first. Because of the large quality factor of the chosen resonator impedance, and therewith the slow decay time of the wakefield, PyHEADTAIL was set up to use the multiturn wake feature. The maximum number of turns for the wake to be taken into account was set to $N_{\text {wake }}=80$ turns, determined by means of a convergence study. Figure 5 summarizes the results. It shows the real (top) and imaginary (bottom) frequencies for the most unstable mode, which is by design an azimuthal mode zero, in the scanned range of $\xi^{(1)}$. PyHEADTAIL results (green crosses) are shown on top of analytical predictions, once including the "full" overlap sum on index $p$ (solid lines), summing up terms from $p=-10^{5}$ to $10^{5}$, and once considering only the most dominant value $p_{0}$ (dashed lines). It can be seen that the full sum shows a very good agreement with the tracking simulations on the full range of $\xi^{(1)}$. The single-peak approximation is close to the PyHEADTAIL results with a discrepancy of less than ten percent in both real and imaginary parts for $-0.4<$ $\xi^{(1)}<-0.2$. It rises, however, to as large as 50 percent in the imaginary part for $\xi^{(1)}>0.1$. For that reason, the scan in second-order chromaticity will be performed at $\xi^{(1)}=$ -0.3 where the approximation works best for both the real and imaginary parts. This is to limit the error on the results of the scan in $\xi^{(2)}$ originating from the single-peak approximation. 


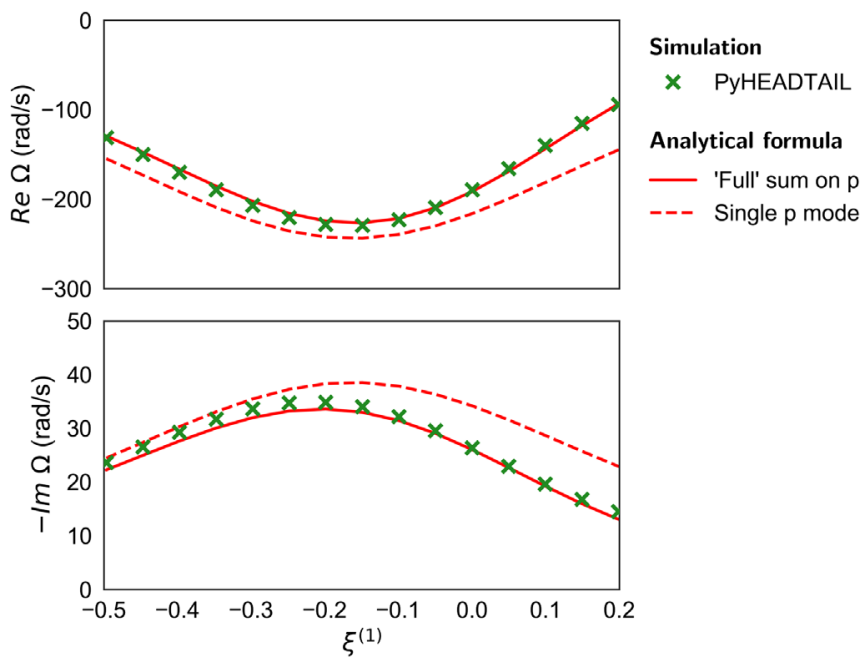

FIG. 5. Real (top) and imaginary (bottom) coherent frequencies of the azimuthal mode zero as a function of $\xi^{(1)}$ (with $\xi^{(2)}=0$ ) for the bunch and machine parameters in Table II. PyHEADTAIL results (green crosses) are compared against analytical calculations. The latter are obtained using either the full overlap sum on index $p$ (solid lines), or only the dominant term $p_{0}$ (dashed lines).

The PyHEADTAIL results of the scan in $\xi^{(2)}$ are displayed in Fig. 6. The plot shows the dependence of the imaginary coherent frequency shift (instability growth rate) on the second-order chromaticity as obtained from tracking (green) when including the betatron frequency spread from $\xi^{(2)}$. Clearly, the stabilization of the mode zero is achieved by introducing a small amount of negative $\xi^{(2)}$ (about -10 units), or a slightly larger amount of positive $\xi^{(2)}$ (about 150 units). Beyond these values, there are no other modes that become unstable given that the scan was performed up to $\left|\xi^{(2)}\right|=1000$. It will be demonstrated in the following with a detailed analysis that the stabilization occurs indeed through the Landau damping mechanism.

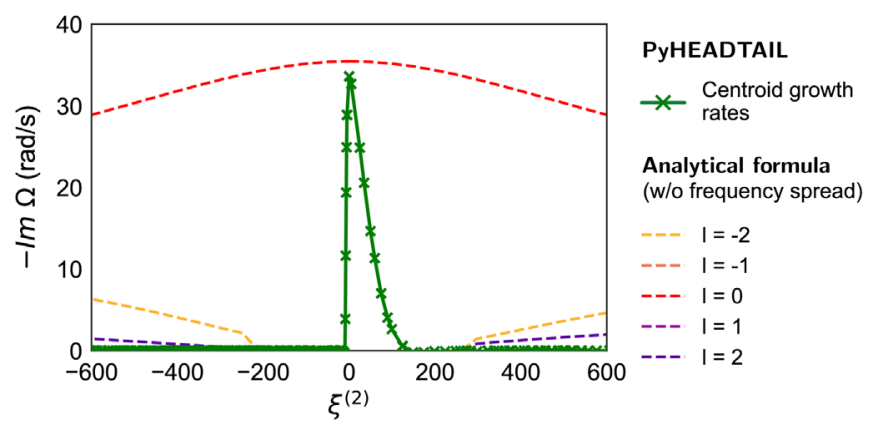

FIG. 6. Dependence of the imaginary coherent frequency shift (growth rate) of the azimuthal mode zero on $\xi^{(2)}$ using the bunch and machine parameters in Table II and $\xi^{(1)}=-0.3$. PyHEADTAIL results (green) include the frequency spread and Landau damping from $\xi^{(2)}$, while analytical calculations (dashed lines) intentionally neglect it to model only the change of effective impedance for comparison.
The reason why a larger amount of second-order chromaticity is required when using a positive sign has already been discussed previously in Refs. [2,7]. It is due to the fact that the specific azimuthal mode has a negative real coherent frequency shift combined with the one-sided frequency spreads (and stability boundary diagrams) that are introduced by $\xi^{(2)}$ as illustrated, e.g., by Fig. 4. In that case, $\xi^{(2)}<0$ provides much more effective Landau damping. Analytical solutions (dashed lines) are shown alongside the tracking results. They deliberately exclude the frequency spread from $\xi^{(2)}$ to remove the effect of Landau damping and to illustrate solely the implications of a change of effective impedance. Unfortunately, this decoupling of the two effects cannot be achieved in a macroparticle tracking code as Landau damping is intrinsically included in this model once nonlinear chromaticity is added. At $\xi^{(2)}=0$, PyHEADTAIL and the analytical prediction are expected to be identical. The small difference is due to the single-peak approximation as already discussed in Fig. 5 (at $\xi^{(1)}=-0.3$ ). The red dashed line indicates that with $\xi^{(2)}$ the growth rate of the mode zero instability is also decreased, but at a much smaller rate compared to PyHEADTAIL. This difference is due to the absence of the frequency spread and Landau damping. Additionally, there are two other modes $(l=-2$ and $l=2)$ that appear according to the Vlasov formalism with a much slower rise time than the mode zero. These modes are also suppressed in PyHEADTAIL. Although not displayed in the figure, the frequency spectra obtained from tracking simulations indicate that the dominant frequency component is always the mode zero which is in agreement with analytical predictions.

In the following, the Vlasov formalism is applied including the betatron frequency spread to make a detailed comparison with the tracking output possible. This is achieved by numerically solving the dispersion relation in Eq. (31), which gives the stability boundary diagram in complex frequency space. The results are only presented for $\xi^{(2)}<0$ here since negative second-order chromaticity provides more effective Landau damping. Stabilization for $\xi^{(2)}>0$ can be shown analogously, but it is less illustrative due to the sharp edge of the frequency spread and of the stability boundary diagrams. The numerical solutions of the dispersion relation are shown in Fig. 7 for six different values of $\xi^{(2)}<0$, increasing in absolute value from top left to bottom right. The plots illustrate the increase of the stability boundary (black line, $-\operatorname{Im} \Omega=0$ ) and hence of the stable area (blue hatched region, $-\operatorname{Im} \Omega \leq 0$ ) in complex frequency space. The instability under consideration is again the head-tail mode zero from Fig. 5 at $\xi^{(1)}=-0.3$. Its coherent frequency shift (red cross) is obtained from PyHEADTAIL simulations. As demonstrated by Fig. 6, the change of the effective impedance induced by $\xi^{(2)}$ is negligible for $\left|\xi^{(2)}\right| \leq 200$ for this specific instability, and 

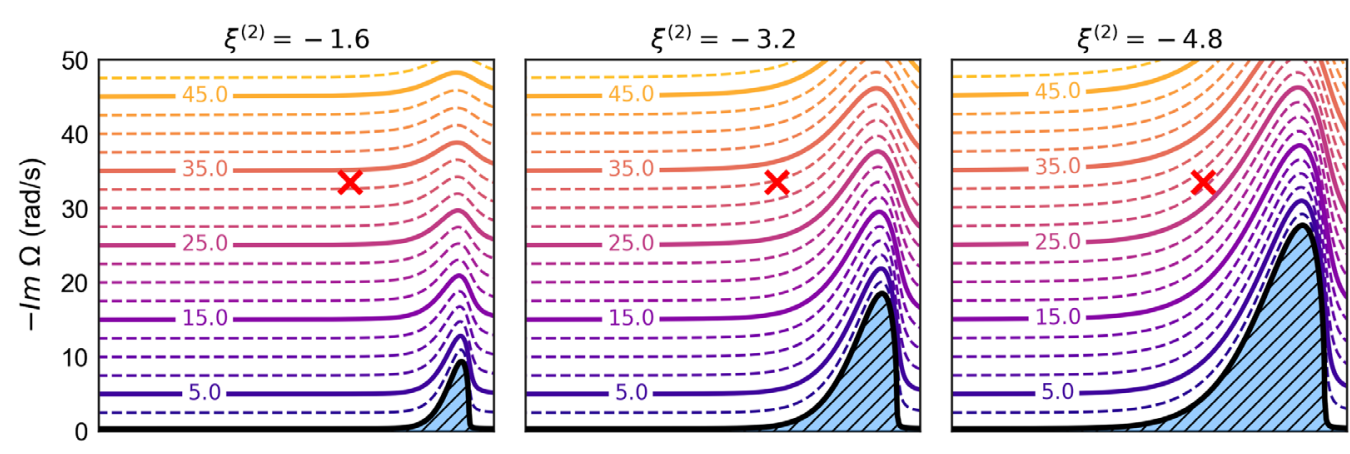

\section{PyHEADTAIL}

$\times$ Coherent frequency

\section{Analytical formula}

1/1/. Stable area

— Stability boundary

Isolines

( $-\operatorname{Im} \Omega=$ const. )
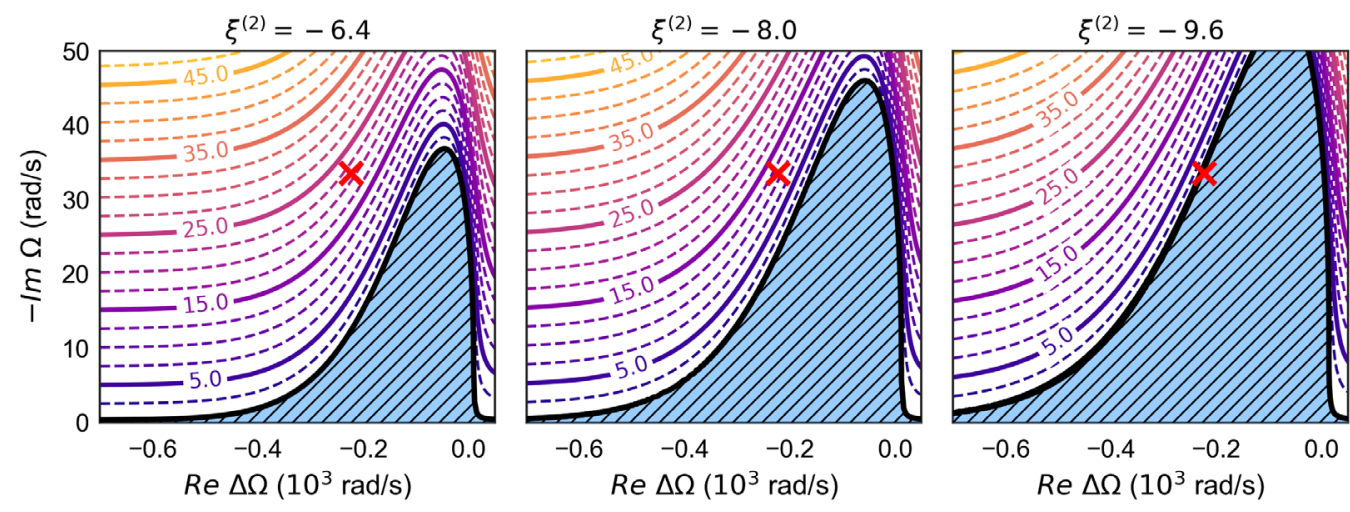

FIG. 7. Stability boundary diagrams in complex frequency space obtained using Eq. (31) are shown for six different values of secondorder chromaticity, with $\left|\xi^{(2)}\right|$ increasing from top left to bottom right. The unstable mode under consideration is marked with the red cross. The stability boundary is given by the black solid line and the area where $-\operatorname{Im} \Omega<0$ is represented by the blue hatched region. The colored lines show a selection of isolines $-\operatorname{Im} \Omega=$ const. and illustrate the distortion of the complex frequency space which becomes stronger with increasing frequency spread from $\xi^{(2)}$.

Landau damping is clearly the dominant effect. As a result, the "unperturbed" coherent frequency can be assumed to be independent of $\xi^{(2)}$. The colored lines in the figure refer to constant values of imaginary frequency shift ( $-\operatorname{Im} \Omega=$ const.) and follow the distortion of the frequency space caused by the spread introduced by second-order chromaticity. They are computed by plugging in different values for $i \varepsilon$ when solving Eq. (31). It can be seen that for real frequency shifts far away from the extent of the spread, the frequency space remains undistorted and the isolines coincide with the ticks shown on the vertical axis.

Using the isolines in Fig. 7, one can read off the effective change, i.e., the decrease, of the imaginary frequency of the unstable mode as a function of frequency spread, or $\xi^{(2)}$. This illustrates the damping process. As the spread increases, the imaginary part of the unstable mode is effectively reduced, meaning that the growth rate of the instability decreases. For $\xi^{(2)} \leq-9.6$, the area of stability becomes large enough as to fully include the unstable mode. At this point the mode is Landau damped. A comparison of the growth rates of the instability between stability diagram theory and PyHEADTAIL tracking simulations is displayed in Fig. 8. The values read off from the stability diagrams and isolines in Fig. 7 are shown in red. The growth rates fitted to the PyHEADTAIL tracking simulations are represented by the green crosses and show an excellent agreement with predictions from theory. Not only the stabilizing threshold for the amount of $\xi^{(2)}$ matches, but also the intermediate stages of $\xi^{(2)}$ show a

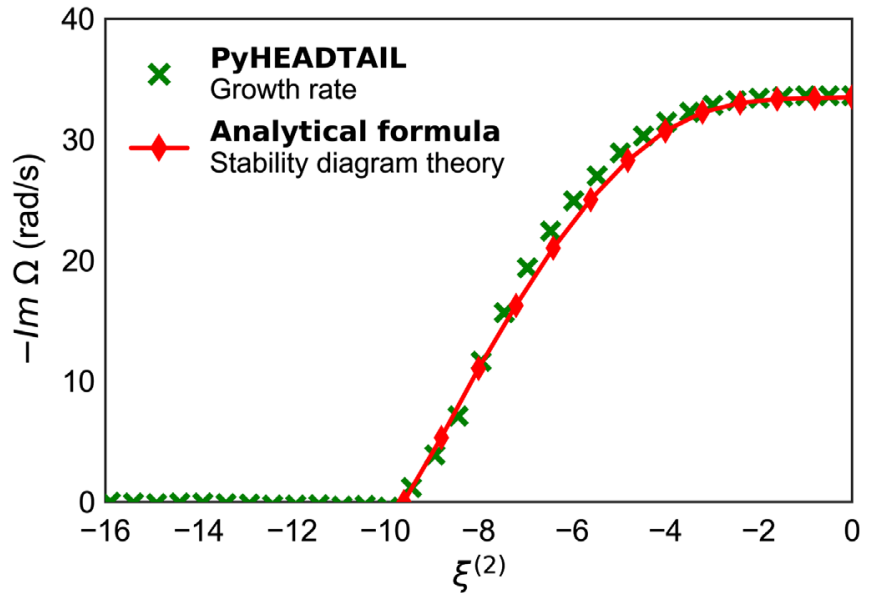

FIG. 8. Stabilization of the head-tail mode zero as a function of $\xi^{(2)}$ for a Gaussian beam. PyHEADTAIL simulations (green crosses) are shown together with analytical predictions computed by means of stability diagram theory (red diamonds). The latter can be deduced from Fig. 7. 
remarkable agreement on the imaginary frequency. This proves that the theory works successfully and that secondorder chromaticity indeed provides Landau damping.

\section{SUMMARY AND CONCLUSIONS}

The existing Vlasov theory on transverse dipole modes has been extended to include the effects of nonlinear chromaticity up to arbitrary order. This new formalism made it possible to confirm the hypothesis that nonlinear chromaticity has two effects on the beam dynamics of transverse coherent modes: (i) it introduces Landau damping thanks to the incoherent betatron frequency spread with longitudinal amplitude, which is present for even orders of chromaticity only, and (ii) it leads to a change of effective impedance. Indeed, the two mechanisms have been identified and studied separately using analytical formulae. This analysis has not been possible for example with tracking simulations as the two effects are closely interlinked in a macroparticle model.

In addition, the theory has been successfully benchmarked up to second-order chromaticity for an airbag model and a Gaussian beam. In the first case, there is no Landau damping due to the missing frequency spread from detuning with longitudinal amplitude. Analytical results have been validated both with a tracking model and a circulant matrix solver which revealed an outstanding agreement. For the Gaussian beam it has been shown that, given the assumption of a strongly-peaked impedance, analytical predictions from stability diagram theory are in excellent agreement with tracking simulations. This proves that detuning with longitudinal amplitude indeed provides Landau damping. The frequency spread can be introduced for example using second-order chromaticity, or, similarly, with an rf quadrupole. This is in accordance with both, experiments and simulations, that were carried out on the rf quadrupole and with second-order chromaticity in the LHC, presented in Refs. [5,7], and confirms the interpretation of these results. All the theory derived for nonlinear chromaticity can also be directly applied to the rf quadrupole.

\section{ACKNOWLEDGMENTS}

The authors would like to thank Elias Métral and Giovanni Rumolo for important inputs to these studies.

\section{APPENDIX A: VLASOV SOLUTION FOR THE AIRBAG BEAM WITH LINEAR CHROMATICITY}

The goal is to demonstrate that for an airbag beam and in case of a purely linear chromaticity, the expression for the coherent frequencies in Eq. (27) reduces to Chao's Eq. (6.188) in Ref. [12]. For a first-order chromaticity $\xi^{(1)}$, the betatron frequency changes as $\Delta \omega_{\beta}=\omega_{\beta, 0} \xi^{(1)} \delta$ and one finds solutions for $\left\langle\Delta \omega_{\beta}\right\rangle_{\phi}(r)$ and $B(r, \phi)$ as given in Eq. (32). It can be shown that the $H_{l}^{p}(r)$ functions reduce to the Bessel functions of the first kind $J_{l}(x)$ under these circumstances

$$
\begin{aligned}
H_{l}^{p}(r) & =\frac{1}{2 \pi} \int_{0}^{2 \pi} e^{i l \phi} e^{-\frac{i \omega^{\prime}}{c} r \cos \phi} e^{-i \xi^{(1) \frac{\omega_{\beta}, 0}{\eta} r} r(1-\cos \phi)} d \phi \\
& =\frac{1}{2 \pi} e^{-i \xi^{(1)} \frac{\omega_{\beta, 0}, 0}{\eta c} r} \int_{0}^{2 \pi} e^{i l \phi} e^{-i\left(\omega^{\prime}-\xi^{(1)} \frac{\omega_{\beta}, 0}{\eta}\right) \frac{r}{c} \cos \phi} d \phi \\
& =i^{-l} e^{-i \xi^{(1) \frac{\omega_{B, 0}, 0}{\eta c} r}} J_{l}\left[\frac{\omega^{\prime}-\omega_{\xi}}{c} r\right] .
\end{aligned}
$$

with $\omega^{\prime}=p \omega_{0}+\omega_{\beta, 0}+l \omega_{s}$ (and $\Omega \approx \omega_{\beta, 0}+l \omega_{s}$ ), and $\omega_{\xi}=\xi^{(1)} \omega_{\beta, 0} / \eta$. By plugging the last result on $H_{l}^{p}(r)$ into Eq. (27), one finds

$\Omega^{(l)}-\omega_{\beta, 0}-l \omega_{s}=-i \frac{N e^{2} c}{2 \omega_{\beta, 0} T_{0}^{2} E} \sum_{p=-\infty}^{\infty} Z_{1}^{\perp}\left(\omega^{\prime}\right) J_{l}^{2}\left(\frac{\omega^{\prime} \hat{z}}{c}-\chi\right)$,

where $\chi=\omega_{\xi} \hat{z} / c$ is the head-tail phase parameter. Equation (A2) is indeed equivalent to Chao's Eq. (6.188) in Ref. [12].

\section{APPENDIX B: EQUIVALENCE OF DISPERSION RELATION TO EXISTING THEORY BY SCOTT BERG AND RUGGIERO}

This section demonstrates that the stability diagram theory developed by Scott Berg and Ruggiero in Ref. [8] for detuning with longitudinal action (which corresponds to a detuning from second-order chromaticity as explained in [7]) is a special solution of Eq. (29) derived in this paper. Scott Berg and Ruggiero's dispersion relation reads [8]

$$
\begin{aligned}
\left(\Delta \Omega_{\operatorname{lin}}^{(l)}\right)^{-1} & =\frac{1}{\widetilde{\mathcal{N}}} \int_{0}^{\infty} \frac{J_{z}^{|l|} \lambda\left(J_{z}\right)}{\Omega^{(l)}-\omega_{\beta}\left(J_{z}\right)-l \omega_{s}} d J_{z}, \\
\widetilde{\mathcal{N}} & =\int_{0}^{\infty} J_{z}^{|l|} \lambda\left(J_{z}\right) d J_{z} .
\end{aligned}
$$

$l$ is the azimuthal mode number of the instability under consideration, and $\lambda$ represents the unperturbed, stationary particle distribution in the longitudinal phase space. $\omega_{\beta}\left(J_{z}\right)=\omega_{\beta, 0}+\Delta \omega_{\beta}\left(J_{z}\right)$ describes the betatron frequency dependence on the longitudinal action $J_{z}$. The latter is related to the longitudinal amplitude $r$ via $r=\sqrt{2 J_{z} \beta_{z}}$. Note that in their paper, Scott Berg and Ruggiero also include a dependence of $\omega_{s}$ on $J_{z}$. This has been ignored here, but could be easily included also in our formalism. Scott Berg and Ruggiero assume in their derivations that: (i) the frequency of the impedance is much smaller than the beam spectrum, and (ii) that the longitudinal wakefield term induced by the transverse wakefield can be ignored. The first approximation basically means that the impedance 
drops out of the equation, similarly to Sec. III B, where a strongly peaked impedance was assumed. The second approximation has also been made in this study when deriving the Vlasov equation in Sec. II A. It is valid as long as synchro-betatron resonance conditions are avoided $\left(\omega_{\beta, 0} \pm l \omega_{s}=n \omega_{0}, \quad\right.$ for $\left.l \in \mathbb{Z}\right)$, and given that the transverse beam sizes are small enough [12].

The derivations made in this paper led to the dispersion relation [Eq. (31)]

$$
\begin{aligned}
\left(\Delta \Omega_{\operatorname{lin}}^{(l)}\right)^{-1} & =\frac{1}{\mathcal{N}} \int_{0}^{\infty} \frac{r g_{0}(r)\left|H_{l}^{p_{0}}(r)\right|^{2}}{\Omega^{(l)}-\omega_{\beta}(r)-l \omega_{s}} d r, \\
\mathcal{N} & =\int_{0}^{\infty} r g_{0}(r)\left|H_{l}^{p_{0}}(r)\right|^{2} d r,
\end{aligned}
$$

where all the functions have been defined in the main text. To demonstrate that this equation is equivalent to Eq. (B1), we start off by approximating the $H_{l}^{p_{0}}(r)$ function. Assuming that the second-order effects from chromaticity accounted for by the phase terms in $H_{l}^{p_{0}}(r)$ can be neglected, and using Eq. (A1), one obtains

$$
\begin{gathered}
H_{l}^{p_{0}} \approx i^{-l} e^{-i \xi^{(1)} \frac{\omega_{\beta, 0}}{\eta c} r} J_{l}\left[\frac{\omega^{\prime}-\omega_{\xi}}{c} r\right], \\
\left|H_{l}^{p_{0}}\right|^{2} \approx\left|J_{l}\left[\frac{\omega^{\prime}-\omega_{\xi}}{c} r\right]\right|^{2}
\end{gathered}
$$

By assumption (i), there is a contribution from the impedance only at low frequencies, meaning that the argument of the Bessel function in the previous equation is small. Hence, $J_{l}(x)$ can be expanded around $x=0$

$$
\begin{aligned}
J_{l}(x) & \approx i^{-l+|l|}\left(\frac{x^{|l|}}{2^{-|l|} \Gamma(1+|l|)}\right) \\
& =i^{-l+|l|}\left(\frac{x^{|l|}}{2^{-|l|}|l| !}\right), \\
\left|J_{l}(x)\right|^{2} & \approx \frac{x^{2|l|}}{2^{-2|l|}|l| !^{2}},
\end{aligned}
$$

since the Gamma function $\Gamma$ is defined as

$$
\Gamma(1+|l|)=|l| !, \quad \text { for }|l| \in \mathbb{N}_{0} .
$$

After changing the integration variable $r \rightarrow J_{z}$, using $r=\sqrt{2 J_{z} \beta_{z}}$, one finds

$$
\begin{aligned}
\left(\Delta \Omega_{\text {lin }}^{(l)}\right)^{-1} & =\frac{C_{l}}{\mathcal{N}} \int_{0}^{\infty} d J_{z} \frac{g_{0}\left(J_{z}\right) J_{z}^{|l|}}{\Omega^{(l)}-\omega_{\beta}\left(J_{z}\right)-l \omega_{s}}, \\
\mathcal{N} & =C_{l} \int_{0}^{\infty} g_{0}\left(J_{z}\right) J_{z}^{|l|} d J_{z},
\end{aligned}
$$

with

$$
C_{l}=\frac{\beta_{z}^{1+|l|}\left[\frac{\omega^{\prime}-\omega_{\xi}}{c} r\right]^{2}}{4|l| !^{2}} .
$$

The constant $C_{l}$ drops out of the equation. After redefining $\mathcal{N}$ accordingly, one indeed reveals Scott Berg and Ruggiero's dispersion relation

$$
\begin{aligned}
\left(\Delta \Omega_{\operatorname{lin}}^{(l)}\right)^{-1} & =\frac{1}{\mathcal{N}} \int_{0}^{\infty} \frac{J_{z}^{|l|} g_{0}\left(J_{z}\right)}{\Omega^{(l)}-\omega_{\beta}\left(J_{z}\right)-l \omega_{s}} d J_{z}, \\
\mathcal{N} & =\int_{0}^{\infty} J_{z}^{|l|} g_{0}\left(J_{z}\right) d J_{z},
\end{aligned}
$$

with $g_{0}\left(J_{z}\right) \equiv \lambda\left(J_{z}\right)$.

[1] A. Grudiev, Radio frequency quadrupole for Landau damping in accelerators, Phys. Rev. Accel. Beams 17, 011001 (2014).

[2] M. Schenk, A. Grudiev, K. Li, and K. Papke, Analysis of transverse beam stabilization with radio frequency quadrupoles, Phys. Rev. Accel. Beams 20, 104402 (2017).

[3] M. Benedikt and F. Zimmermann, Future Circular Colliders, CERN Technical Report No. CERN-ACC-20150164, 2015.

[4] J. Gareyte, J. P. Koutchouk, and F. Ruggiero, Landau damping, dynamic aperture and octupoles in LHC, CERN Technical Report No. CERN-LHC-Project-Report-91, 1997.

[5] M. Schenk, X. Buffat, L. Carver, A. Grudiev, K. Li, A. Maillard, E. Métral, and K. Papke, in Proc. of International Particle Accelerator Conference (IPAC'17), Copenhagen, Denmark, 2017, International Particle Accelerator Conference No. 8 (JACoW, Geneva, 2017), p. 2516.

[6] O. S. Brüning, P. Collier, P. Lebrun, S. Myers, R. Ostojic, J. Poole, and P. Proudlock, LHC Design Report, CERN Yellow Reports: Monographs (CERN, Geneva, 2004).

[7] M. Schenk, X. Buffat, L. R. Carver, R. De Maria, K. $\mathrm{Li}$, and E. Métral, Experimental stabilization of transverse collective instabilities in the LHC with second order chromaticity, Phys. Rev. Accel. Beams 21, 084401 (2018).

[8] J. S. Berg and F. Ruggiero, in Proceedings of the Particle Accelerator Conference, Vancouver, BC, Canada, 1997 (IEEE, New York, 1997), Vol. 2, p. 1712.

[9] V. Danilov and E. Perevedentsev, Feedback system for elimination of the transverse mode coupling instability, Nucl. Instrum. Methods Phys. Res., Sect. A 391, 77 (1997).

[10] X. Buffat, Transverse beams stability studies at the Large Hadron Collider, Ph.D. thesis, SB, Lausanne, 2015.

[11] E. Métral, T. Argyropoulos, H. Bartosik, N. Biancacci, X. Buffat, J. F. E. Muller, W. Herr, G. Iadarola, A. Lasheen, K. Li et al., Beam instabilities in hadron synchrotrons, IEEE Trans. Nucl. Sci. 63, 1001 (2016). 
[12] A. Chao, Physics of Collective Beam Instabilities in High Energy Accelerators, Wiley Series in Beam Physics and Accelerator Technology (Wiley, New York, 1993).

[13] T. Suzuki, Fokker-planck theory of transverse mode coupling instability, Part. Accel. 20, 79 (1986).

[14] R. R. Lindberg, Fokker-Planck analysis of transverse collective instabilities in electron storage rings, Phys. Rev. Accel. Beams 19, 124402 (2016).
[15] L. D. Landau, On the vibrations of the electronic plasma, Zh. Eksp. Teor. Fiz. 16, 574 (1946) [J. Phys. USSR 10, 25 (1946)].

[16] G. Rumolo and F. Zimmermann, Electron cloud simulations: beam instabilities and wakefields, Phys. Rev. Accel. Beams 5, 121002 (2002).

[17] R. Bartolini and F. Schmidt, A computer code for frequency analysis of nonlinear betatron motion, CERN Technical Report No. SL-Note-98-017-AP, 1998. 